\title{
The relation between lesions and localization of sources of slow biphasic complexes in encephalitis
}

\author{
Massimo Valerio, Stefano Rivera, Luca Mesin \\ Mathematical Biology \& Physiology, Department of Electronics and Telecommunications, Politecnico di Torino, Turin 10129, \\ Italy.
}

Correspondence to: Prof. Luca Mesin, Mathematical Biology \& Physiology, Department of Electronics and Telecommunications, Politecnico di Torino, Corso Duca degli Abruzzi 24, Turin 10129, Italy. E-mail: luca.mesin@polito.it

How to cite this article: Valerio M, Rivera S, Mesin L.The relation between lesions and localization of sources of slow biphasic complexes in encephalitis. Neuroimmuno/ Neuroinflammation 2021;8:42-9. http://dx.doi.org/10.20517/2347-8659.2020.30

Received: 11 Apr 2020 First Decision: 28 May 2020 Revised: 12 Jun 2020

Accepted: 28 Jun 2020 Fisrt online: 15 Aug 2020 Published: 21 Mar 2021

Academic Editor: Mercè Falip Copy Editor: Cai-Hong Wang Production Editor: Jing Yu

\begin{abstract}
Slow biphasic complexes (SBC) were found in the electroencephalogram (EEG) of patients with inflammations of the brain. We have developed an automated method to identify them and proved that they represent a sensitive marker of the severity of encephalitis. Here we focus on another property of SBCs, i.e., the localization of their sources. We present two encephalitic patients, showing lesions in the magnetic resonance images, which are either spread in the brain or focused on the left hemisphere, respectively. Applying a source localization algorithm to the identified SBCs, we found either a diffused or a left-focused distribution, respectively. This result further suggests a relation between neuroinflammation and appearance of SBCs, indicating that their distribution reflects in part the localization of brain lesions. This promising result extends the information that can be extracted from EEG, promoting the reduction of expensive or invasive measurements in encephalitic patients.
\end{abstract}

Keywords: EEG, encephalitis, slow biphasic complex, source localization

\section{INTRODUCTION}

Encephalitis is an inflammatory process of the cerebral parenchyma associated with neurological dysfunctions $^{[1]}$, which requires prompt diagnosis and intervention ${ }^{[2]}$. It is caused mainly by infectious diseases or immune disorders, cancer, and vascular problems ${ }^{[3,4]}$. It can have different progressions (acute,

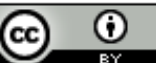

(C) The Author(s) 2021. Open Access This article is licensed under a Creative Commons Attribution 4.0 International License (https://creativecommons.org/licenses/by/4.0/), which permits unrestricted use, sharing, adaptation, distribution and reproduction in any medium or format, for any purpose, even commercially, as long as you give appropriate credit to the original author(s) and the source, provide a link to the Creative Commons license, and indicate if changes were made.

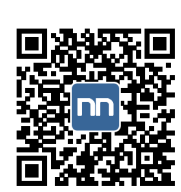


subacute, or chronic) and patients frequently report neurological sequelae ${ }^{[3,4]}$. The incidence of infectious encephalitis is estimated at 1.5-7 cases per 100,000 inhabitants/year in the world ${ }^{[5]}$, and 10.09 cases per 100,000 in Italian infants ${ }^{[4]}$. Encephalitis is a serious problem requiring hospitalization and giving a significant economic burden on society ${ }^{[6]}$.

In more than $50 \%$ of cases the etiological cause is unknown and patients are admitted with non-specific symptoms at the time of presentation ${ }^{[7]}$. However, the main manifestations are brain suffering and/or altered state of consciousness, possibly in addition to fever, focal neurological deficits, epileptic seizures, abnormalities in the electroencephalogram (EEG) or neuroimaging, and cerebrospinal fluid (CSF) pleiocytosis. After a rapid evaluation of basic vital functions, serological and instrumental tests, empirical urgent therapy is usually adopted for symptomatic patients, in association with antiviral, antibiotic, and steroid drugs ${ }^{[8]}$. Prognosis is difficult to evaluate, mainly due to the multiple possible aetiologies (for example, in the case of herpes simplex encephalitis, which is the most common one, mortality in the range of 5\%-20\% was documented for patients treated with antiviral, $70 \%$ in those who did not receive treatment) ${ }^{[9]}$.

The EEG has a fundamental role in the diagnostic framework. Different aetiologies of encephalitis were found to be associated with specific EEG patterns: for instance, triphasic waves are pathognomonic of hepatic encephalopathy ${ }^{[10]}$ and lateralized periodic discharges or periodic lateralized epileptiform discharges are found in herpetic encephalopathy ${ }^{[11]}$. Moreover, stage II of subacute sclerosing panencephalitis is characterized by bilaterally symmetrical and synchronous generalized, stereotyped high amplitude delta waves, called Radermacker or "R" complexes ${ }^{[12]}$. Here we are interested in a specific EEG element, called slow biphasic complex (SBC), described as identical in the first part to the " $\mathrm{R}$ " complex even if it has different spatial and temporal properties ${ }^{[13]}$. SBC has been described as associated with the inflammatory processes of the central nervous system ${ }^{[13-17]}$. We have recently proposed an automated method to identify SBCs in an EEG trace, opening the possibility of quantifying them and investigating their origin. In particular, we have demonstrated that the number and amplitude of complexes reflect the severity of the inflammation in pediatric encephalitic patients ${ }^{[14]}$. Moreover, we have proposed to integrate information from different features of SBC to improve the diagnosis ${ }^{[18]}$.

Herein, we focus on the relation between the location of SBCs and brain lesions found in magnetic resonance images (MRI). Methods for EEG source detection are applied to the identified SBCs to localize the brain areas producing them. This could be a promising tool to investigate the topography of inflammatory activity ${ }^{[19]}$. We report the application of this method in two specific cases.

\section{CASE REPORT}

We applied our processing to the EEG recorded from two patients also considered in a previous paper ${ }^{[14]}$ (to which the reader can refer for details on EEG recordings), for which MRIs were also available. For each patient, we considered EEG data recorded close to the day in which the MRI was acquired. The two patients were very different, the first showing diffused lesions due to acute disseminated encephalomyelitis (ADEM) and the second with inflammatory processes, caused by infectious etiology, focused in one hemisphere. In this section, we first introduce the processing methods; then the two cases are discussed.

\section{Methods}

An algorithm we introduced before was applied to identify the $\mathrm{SBCs}^{[14]}$. Then, a method for source localization was used to identify the brain areas involved in the production of the complexes. These locations were compared to those of lesions identified in the MRIs by an expert neuro-radiologist.

Identification of slow biphasic complexes

SBCs were identified by the method described in a previous paper ${ }^{[14]}$. In brief, each EEG trace was 
processed with a set of match filters, each comparing the signal with a scaled version of a prototype biphasic waveform. The identified complexes were then automatically reviewed, excluding outliers and waveforms showing repetitive firings, as some waveforms could have a shape similar to that of an SBC, but they could not satisfy some properties indicated in previous publications ${ }^{[14,17,18]}$.

\section{Source localization}

Source localization in EEG refers to the detection of the sources inside the brain that generate the electrical activity acquired on the scalp. When the available electrodes are in a small number (as in our cases, in which either 12 or 18 channels were available for the two cases, respectively), the source detection may have low accuracy ${ }^{[20]}$, but can provide useful information on the brain areas that are most involved in the inflammatory activity.

From the mathematical point of view, the dipoles inside the brain that produce a scalp potential that best fits the original data are sought. The problem can be written as follows:

$\mathrm{M}=\mathrm{GD}+\mathrm{n}$

where each row of the matrix M contains a measured EEG, $G$ is the Lead-Field matrix that describes the response of the activation of $\mathrm{N}$ different dipoles, whose level of activity (collected in $\mathrm{D}$ ) should be estimated, and $\mathrm{n}$ is an additive noise, assumed spatially and temporally white. Different methods have been proposed in the literature to solve this problem ${ }^{[19,21]}$. In this study, the minimum norm estimation (MNE) was used $^{[22]}$. It searches for the solution with minimum power, by minimizing the following regularized functional

$U(\mathrm{D})=\|\mathrm{M}-\mathrm{GD}\|^{2}+a\|\mathrm{D}\|^{2}$

where $\alpha$ is a regularization parameter to constrain the power of the solution (chosen in this study to be equal to the mean of the eigenvalues of $\mathrm{G}^{\mathrm{T}} \mathrm{G}$ divided by 2,500; however the estimation was stable to a variation of $\alpha$ by an order of magnitude). It brings to the following solution to recover the sources:

$D_{M N E}=\left(G^{T} G+a I_{N}\right)^{-1} G^{T} M$

where $\mathrm{I}_{\mathrm{N}}$ is the identity matrix of dimension $\mathrm{N} \times \mathrm{N}$.

\section{Localization of sources of $S B C s$}

The waveforms of interest are concentrated in a low-frequency band. After visual inspection of the portions containing SBCs, each EEG trace was then band-pass filtered between 0.5 and $5 \mathrm{~Hz}$ (Chebychev filter of order 6 of type I), and the common-mode was removed. This filter provided clean EEG traces, focusing the source detection mostly on the waveforms of interest. Half second long windows centered on the identified SBCs were concatenated to generate the rows of matrix M in equation (1). Then, MNE (FieldTrip implementation ${ }^{[23]}$ ) provided a discrete brain model made of equivalent current dipole sources, containing the mean activation over time for each source location. The same procedure was applied to EEG data with the same duration obtained concatenating windows not including SBCs, to estimate the average background activity. The difference between the medians of dipole intensities during SBC onsets and in the background was then investigated (checking significant differences with the Wilcoxon rank-sum test for equal medians, with significance level $P=0.001$ ).

\section{First case}

A 4-year-old subject was considered. At the time of the presentation, the patient presented with fever, 

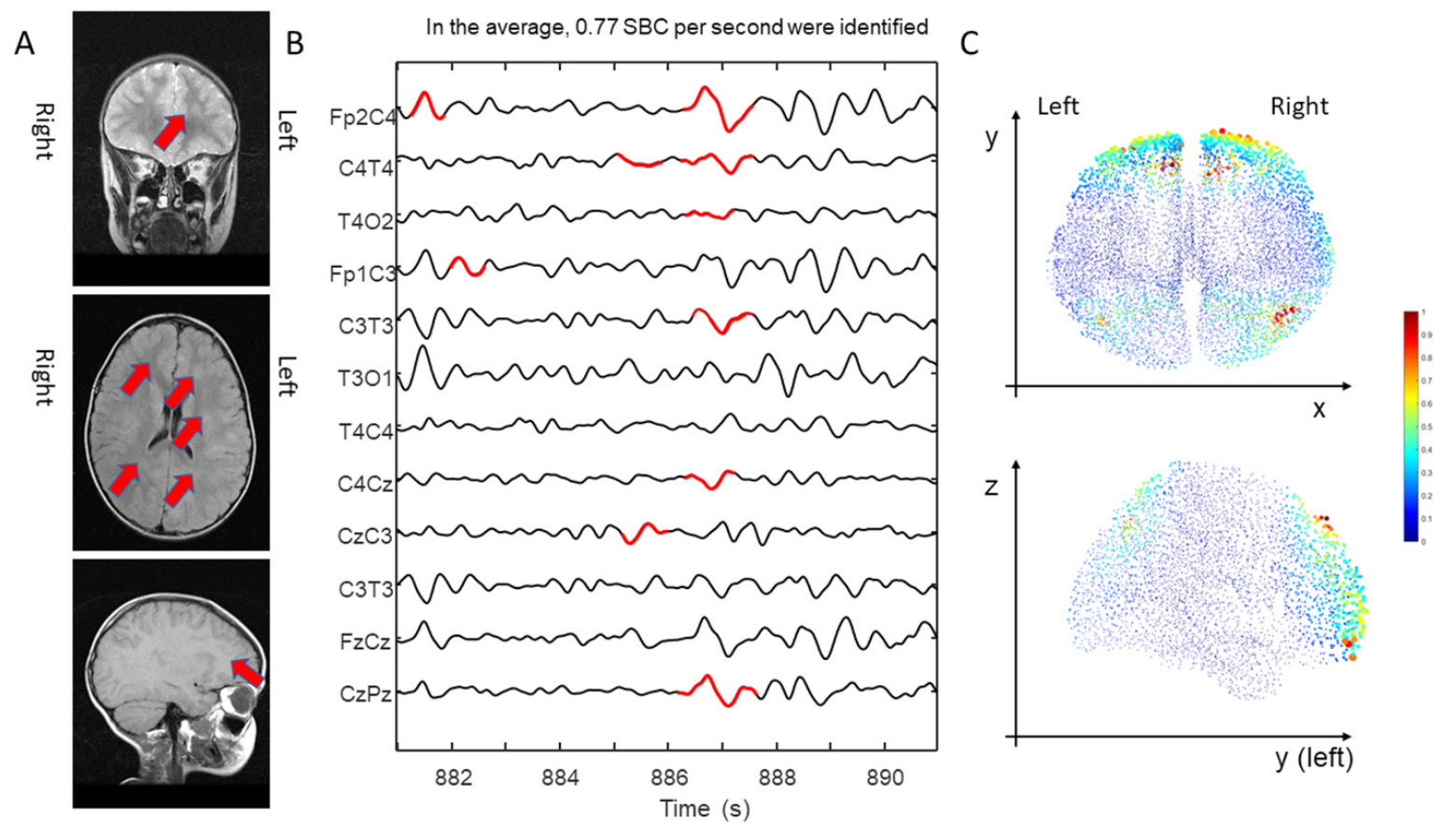

Figure 1. Coronal, axial, and sagittal magnetic resonance images sections of the brain for the first case. The lesions are highlighted with red arrows (radiological convention) (A); a portion of electroencephalogram (filtered between $0.5 \mathrm{and} 5 \mathrm{~Hz}$ ) with an indication of the identified slow biphasic complexes (SBC) in red color (B); the normalized mean power of the difference between the intensities of the sources of the signal during SBCs activation and background (neurological convention) (C)

headache, lower back pain, and somnolence. The symptoms, after a temporary regression with nonsteroidal anti-inflammatory drugs, deteriorated in the following days leading to confusion, plaintive, and drowsiness and patient was hospitalized. Empirical therapy was administered with antibiotics, antiviral, and steroid therapy. Lumbar puncture was performed which demonstrated an elevated CSF pressure, but laboratory tests (i.e., physical chemical test and cell counting) were negative. Blood chemistry tests showed a mild increase in white cells and high inflammation indexes.

The EEG performed at the onset showed a severe widespread brain suffering from SBCs on the frontal areas. The first brain MRI showed an ADEM-compatible result, with cortico-subcortical lesions prevalently observable in the frontal lobe (bigger on the left), medial temporal cortex, and basal ganglia.

We processed an EEG trace acquired the day before the MRI registration. The results are shown in Figure 1. The MRI is shown on the left with an indication of the main lesions. A portion of EEG is shown in the center, with the identified SBCs super-imposed in red color. The localization of SBC sources (emerging from the background) is shown on the right. The intensity of dipole sources during SBC activity resulted significantly different from the background (higher, actually) in 99\% of cases. An important increase in intensity can be appreciated for the dipoles in the frontal area and the parietal-occipital lobe. The activity is quite spread across the two hemispheres.

During hospitalization, there was a slow improvement in clinical and instrumental examinations. The patient after a month was dismissed from the hospital with ADEM diagnosis and a schedule of follow-up.

\section{Second case}

A 10-year-old subject was considered. In the beginning, the child was diagnosed with a rubella type rash that after 5 days evolved presenting neurological symptoms with photophobia and drowsiness alternating 

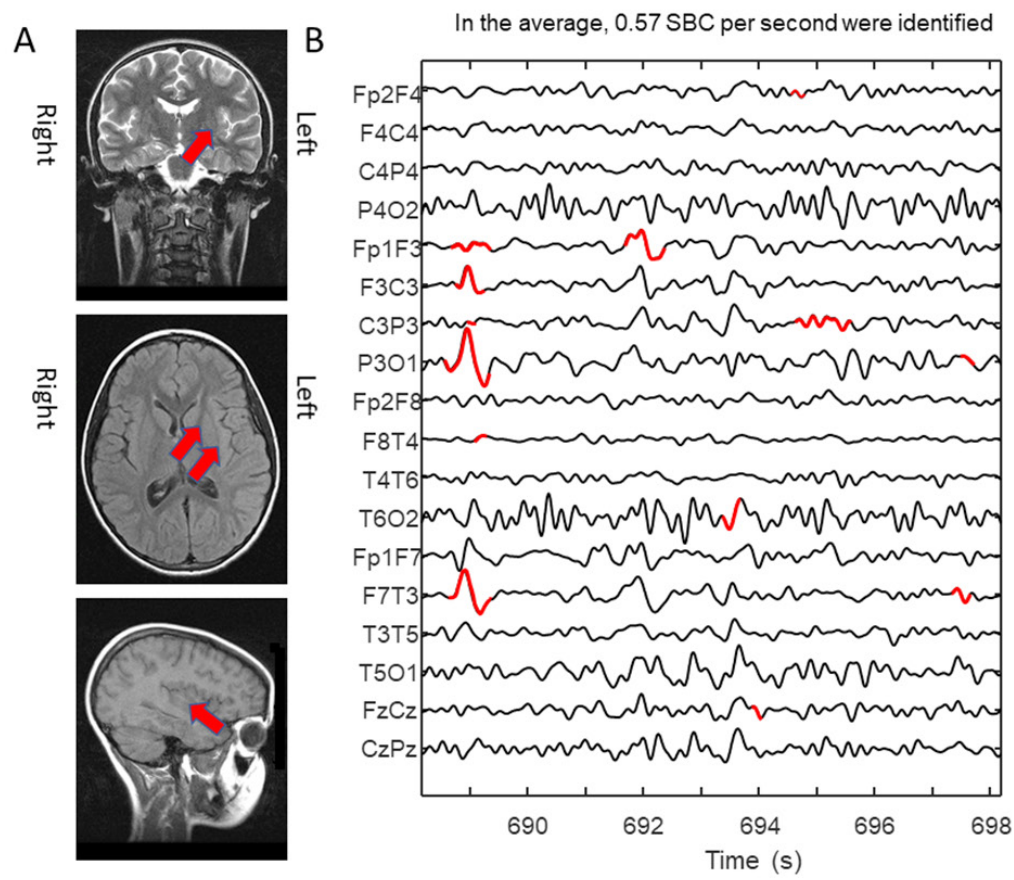

C
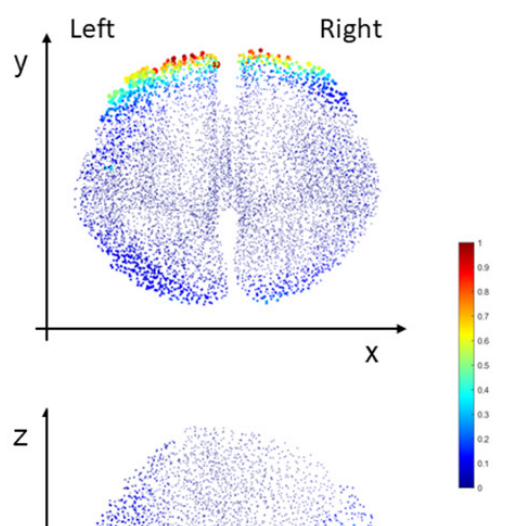

Figure 2. Coronal, axial, and sagittal magnetic resonance images sections of the brain for the second case. The lesions are highlighted with red arrows (radiological convention) (A); a portion of electroencephalogram (filtered between $0.5 \mathrm{and} 5 \mathrm{~Hz}$ ) with an indication of identified slow biphasic complexes (SBC) (B); the normalized mean power of the difference between the intensities of the sources of the signal during SBCs activation and background (neurological convention) (C)

with psychomotor agitation. The results of serology and bacteriological tests showed a slight increase in inflammatory parameters, in particular of lymphocytes. The examination of the CSF extracted by a lumbar puncture reported increased cellularity. Due to the worsening condition, the child was transferred to our tertiary children's center to be admitted to the intensive care unit. The adopted empirical therapy consisted of a triad of drugs formed by the antibiotic, steroid, and antiviral drugs (as in the previous case), with the addition of antifungal therapy.

The EEG traces initially showed a very slow widespread electrical activity that after a few days has been focused on the left hemisphere, in particular on the frontal areas. The MRI, acquired after the admission of the patient in the intensive care unit, showed lesions in particular in the flair sequence in the areas parasagittal frontal and mesial - insular temporal in the left hemisphere.

We processed an EEG trace acquired two days before the MRI registration. The results are shown in Figure 2. The MRI is shown on the left, a portion of EEG in the center, and the localization of SBC sources (emerging from background) on the right. Also, in this case, the intensity of dipole sources during SBC activity was significantly higher than the background in $99 \%$ of cases. An important increase in intensity can be appreciated for the dipoles in frontal area (mainly on the left) and a superficial portion of the occipital lobe. Most activity is found in the left hemisphere.

Afterward, the clinical situation improved, but the child showed a deficit in the right side of the body. She was dismissed with a diagnosis of encephalitis during rubella infection.

\section{DISCUSSION}

In the literature, several studies recognized the possibility to identify the etiological causes of different pathologies based on the EEG ${ }^{[24,25]}$. This allows us to advance diagnostic hypotheses and to outline 
predictive factors, establishing different outcomes. Early EEG alterations suggest a negative prognosis, supporting the use of aggressive anti-inflammatory neuroprotection therapies ${ }^{[26,27]}$.

SBCs have been observed in patients with brain inflammations ${ }^{[13,15,28,29]}$. We have developed an automated processing method to identify SBCs and we have shown in previous studies a good correlation between their onset and the severity of encephalitis ${ }^{[14,18]}$. Here we have focused on the distribution of the sources of SBCs and their relation with the lesions identified in MRIs.

Two different cases are discussed. In the first, lesions were widespread in the brain, whereas in the second they were more focused on the left hemisphere. Sources of SBCs were also found more widespread in the first case and predominantly on the left hemisphere in the second, indicating that the localization of SBC sources can provide some insights on the location of the lesions.

However, we should notice that SBC sources were identified quite superficial, mostly in frontal location and not exactly in the sites of the lesions. Notice that the low-frequency activity investigated in this study (predominantly in the delta range and increased in our patients, due to brain suffering from encephalitis) is larger in the frontal lobe, even during periods in which SBCs are not present. However, the sources of SBCs are significantly larger than those producing background activities, indicating that the predominant identification of SBC sources in the frontal lobe is not a bias.

Possibly, our results could be biased by both the source localization algorithm and the surface EEG technique in general, which emphasizes cortical contributions. Indeed, the activity of cortical neurons is recorded with a larger amplitude than that of deeper sources (possibly, even appearing under the noise level or covered by the synchronous cortical activity of areas also affected by the inflammation). Consider also that a small number of electrodes was used in our clinical recordings, hampering the identification of deep sources ${ }^{[20]}$. Moreover, lesions affect the activity of cortical neurons connected to the inflamed ones: these connected neurons could also be far apart from the lesions. Thus, it could be possible to observe altered activity not only in a contiguous area, but also distant from the lesions, i.e., produced by cortical neurons in connection with the focal area of inflammation, but located far apart (reflecting a well-known difference between anatomical and functional correlates in the brain). Notice also that, if the area of inflammation is located in the white matter, it affects only axons and action potentials propagating along them are less visible from EEG than post-synaptic potentials. However, as mentioned above, even if the exact locations of the lesions are not easy to be identified, our results suggest that the spread of the lesions and a possible asymmetry can be found (indeed, in the first case, the identified SBC sources are widespread and, in the second case, SBC sources are predominantly found in the same hemisphere in which lesions are located).

Further work is suggested to deepen the promising results found in this pilot study on a few patients. In particular, using a high-density system for EEG acquisition could allow us to better locate the sources of SBCs, to be correlated with MRI results ${ }^{[30]}$. Moreover, the use of functional MRI in synchronous with EEG registration ${ }^{[31]}$ could help in investigating better SBC sources.

As EEG acquisition is cheaper and faster than MRI recording, the possible confirmation of the relation between SBC sources location and lesions could have relevance in the clinical practice. Specifically, EEG could support the follow-up of the patient, e.g., with daily monitoring of the effect of the treatment on the possible reduction of the lesions. This method, if confirmed in an extended study, could support the clinician in rapid diagnosis, allowing the fast implementation of specific therapy to improve the prognosis and simple monitoring of the progress of the patient. 


\section{DECLARATIONS}

\section{Acknowledgments}

The authors are grateful to Prof. B. Vitiello and Prof. G. Capizzi who provided access to the clinical data discussed in this work.

\section{Authors' contributions}

Data selection and interpretation of results: Valerio $\mathrm{M}$

Localization of SBC sources: Rivera S

Identification of SBCs and supervision of the other activities: Mesin L

\section{Availability of data and materials}

Data will not be shared.

\section{Financial support and sponsorship}

None.

\section{Conflicts of interest}

All authors declared that there are no conflicts of interest.

\section{Ethical approval and consent to participate}

A consent for the examination and data processing was obtained from the parents of the patients.

\section{Consent for publication}

Not applicable.

\section{Copyright}

(c) The Author(s) 2021.

\section{REFERENCES}

1. Tunkel AR, Glaser CA, Bloch KC, Sejvar JJ, Marra CM, et al. The management of encephalitis: clinical practice guidelines by the Infectious Diseases Society of America. Clin Infect Dis 2008;47:303-27.

2. Piquet AL, Cho TA, The Clinical Approach to Encephalitis. Curr Neurol Neurosci Rep 2016;16:45.

3. Kneen R, Michael BD, Menson E, Mehta B, Easton A, et al. Management of suspected viral encephalitis in children - Association of British Neurologists and British Paediatric Allergy, Immunology and Infection Group national guidelines. J Infect 2012;64:449-77.

4. Barbadoro P, Marigliano A, Ricciardi A, D'Errico MM, Prospero E. Trend of hospital utilization for encephalitis. Epidemiol Infect 2012;140:753-64.

5. Boucher A, Herrmann JL, Morand P, Buzelé R, Crabol Y et al. Epidemiology of infectious encephalitis causes in 2016. Med Mal Infect 2017;47:221-35.

6. Vora NM, Holman RC, Mehal JM, Steiner CA, Blanton J, et al. Burden of encephalitis-associated hospitalizations in the United States, 1998-2010. Neurology 2014;82:443-51

7. Glaser CA, Honarmand S, Anderson LJ, Schnurr DP, Forghani B, et al. Beyond viruses: clinical profiles and etiologies associated with encephalitis. Clin Infect Dis 2006;43:1565-77.

8. Thompson C, Kneen R, Riordan A, Kelly D, Pollard AJ, et al. Encephalitis in children. Arch Dis Child 2012;97:150-61.

9. Zuo XZ, Tang WJ, Chen XY, Huang W. A review with comments on herpes simplex encephalitis in adults. Neuroimmunol Neuroinflammation 2017;4:24-7.

10. Emmady PD, Murr N. EEG, Triphasic Waves. StatPearls Publishing; 2020.

11. Lin L, Drislane F. Lateralized Periodic Discharges. J Clin Neurophysiol 2018;35:189-98.

12. Andraus MEC, Andraus CF, Alves-Leon SV. Periodic EEG patterns: importance of their recognition and clinical significance. Arq Neuropsiquiatr 2012;70:145-51.

13. Beaumanoir A, Magistris M, Nahory A. Sporadic slow biphasic complex: description and clinical correlations. Electroencephalogr Clin Neurophysiol 1985;61:142.

14. Mesin L, Valerio M, Beaumanoir A, Capizzi G. Automatic identification of slow biphasic complexes in EEG: an effective tool to detect encephalitis. Biomed Phys Eng Express 2019;5:045006. 
15. Beaumanoir A, Grioni D, Kullmann G, Tiberti A, Valseriati D. EEG anomalies in the prodromic phase of Rasmussen's syndrome. Report of two cases. Neurophysiol Clin 1997;27:25-32.

16. Maciel CB, Hirsch LJ. Definition and classification of periodic and rhythmic patterns. J Clin Neurophysiol 2018;35:179-88.

17. Gatti A, Guarneri M, Motto CA, Vigliano P, Beaumanoir A. Slow biphasic complex: electro-clinical considerations. Ital J Neurol Sci 1997;18:99.

18. Mesin L, Valerio M, Capizzi G. Automated diagnosis of encephalitis in pediatric patients using EEG rhythms and slow biphasic complexes. Phys Eng Sci Med 2020; [published online ahead of print, 2020 Jul 21].

19. Grech R, Cassar T, Muscat J, Camilleri K, Fabri SG, et al. Review on solving the inverse problem in EEG source analysis. J Neuroeng Rehabil 2008;5:25.

20. Vatta F, Bruno P, Inchingolo P. Accuracy of EEG source reconstruction in the presence of brain lesions: modelling errors and surface electrodes' placement. Biomed Sci Instrum 2002;38:423-8

21. Jatoi MA, Kamel N, Saeed A, Malik AS, Faye I, et al. A survey of methods used for source localization using EEG signals. Biomed Sig Proc Control 2014;11: 42-52.

22. Dale AM, Sereno MI. Improved localizadon of cortical activity by combining EEG and MEG with MRI cortical surface reconstruction: a linear approach. J Cogn Neurosci 1993;5:162-76.

23. Oostenveld R, Fries P, Maris E, Schoffelen JM. FieldTrip: open source software for advanced analysis of MEG, EEG, and invasive electrophysiological data. Comput Intell Neurosci 2011;2011:156869

24. Appavu B, Riviello JJ. Electroencephalographic patterns in neurocritical care: pathologic contributors or epiphenomena? Neurocrit Care 2018;29:9-19.

25. Yildirim M, Konuskan B, Yalnizoglu D, Topaloglu H, Erol I, et al. Electroencephalographic findings in anti-N-methyl-d-aspartate receptor encephalitis in children: a series of 12 patients. Epilepsy Behav 2018;78:118-23.

26. Sutter R, Kaplan PW, Valença M, De Marchis GM. EEG for diagnosis and prognosis of acute nonhypoxic encephalopathy: history and current evidence. J Clin Neurophysiol 2015;32:456-64.

27. Mohammad SS, Soe S, Pillai SC, Nosadini M, Barnes EH, et al. Etiological associations and outcome predictors of acute electroencephalography in childhood encephalitis. Clin Neurophysiol 2016;127:3217-24.

28. Tanoue K, Oguni H, Nakayama N, Sasaki K, Ito Y, et al. Focal epileptic spasms, involving one leg, manifesting during the clinical course of west syndrome (WS). Brain Dev 2008;30:155-9.

29. Chen L, Zhu M, Zhou H, Liang J. Clinical study of West syndrome with PS and late-onset epileptic spasms. Epilepsy Res 2010;89:82-8.

30. Olivieri G, Contaldo I, Ferrantini G, Musto E, Scalise R, et al. Autoimmune encephalopathies in children: diagnostic clues and therapeutic challenges. Neuroimmunol Neuroinflammation 2016;7:147

31. Vitali P, Di Perri C, Vaudano AE, Meletti S, Villani F. Integration of multimodal neuroimaging methods: a rationale for clinical applications of simultaneous EEG-fMRI. Funct Neurol 2015;30:9-20. 